\title{
Timing of Nonculprit Percutaneous Coronary Intervention after ST-Elevation Myocardial Infarction
}

\author{
Joshua H. Arnold ${ }^{a, b}$ Tamir Bental ${ }^{a, b} \quad$ Gabriel Greenberga, ${ }^{a}$ \\ Hana Vaknin-Assa ${ }^{a, b}$ Ran Kornowskia, ${ }^{a}$ Leor Perl ${ }^{a, b}$ \\ ${ }^{a}$ Department of Cardiology, Rabin Medical Center, Petach Tikva, Israel; ${ }^{b}$ Faculty of Medicine, Tel Aviv University, Tel \\ Aviv, Israel
}

\section{Keywords}

Primary percutaneous coronary intervention - ST-elevation myocardial infarction · Culprit artery

\begin{abstract}
Introduction: Complete revascularization of ST-elevation myocardial infarction (STEMI) patients with multivessel disease (MVD) has recently shown to reduce risk of adverse cardiovascular events, including cardiovascular death. Optimal timing of revascularization of nonculprit lesions remains controversial. We aimed to measure cardiac outcomes related to duration between primary percutaneous coronary intervention $(\mathrm{pPCl})$ of the culprit lesion and staged $\mathrm{PCl}(\mathrm{sPCl})$ of nonculprit lesions. Methods: From a prospectively collected consecutive registry of 3,002 patients treated for STEMI by $\mathrm{pPCl}, 1,555$ patients with $\mathrm{MVD}$ requiring $\mathrm{SPCl}$ were identified. Patients were placed into quartiles of duration to $\mathrm{SPCl}$ : 0-7 days (Q1), 7-22 days (Q2), 22-42 days (Q3), >42 days (Q4), excluding those who had complete revascularization at the index event. Major adverse cardiac events (MACEs) included all-cause mortality, myocardial infarction, target vessel revascularization, and coronary artery bypass surgery. Cox regression and propensity score matching were performed correcting for confounding factors. Results: The av-
\end{abstract}

erage age at presentation was $65.7 \pm 11.5$ years. 333 were female (21.4\%). Mean time between $\mathrm{pPCl}$ and $\mathrm{sPCl}$ was 28.3 days ( \pm 24.8$)$. Rates of MACE were Q1 - 16.5\%, Q2 - 21.2\%, Q3 - 25.8\%, and Q4 - 30.1\% (log-rank <0.001). Following regression analysis, SPCl remained an independent risk factor for MACE (hazard ratio $[\mathrm{HR}]=1.226[95 \%$ confidence interval $\{C l\}: 1.129-1.331, p<0.001])$. There was no association between the time interval up to $\mathrm{SPCl}$ with all-cause death $(\mathrm{HR}=1.022$ [95\% Cl: $0.925-1.129, p=0.671])$. Conclusions: Patients with MVD are at increased risk of experiencing MACE after revascularization of nonculprit vessels with increasing time delay between $\mathrm{pPCl}$ and $\mathrm{sPCl}$.

๑) 2021 S. Karger AG, Basel

\section{Introduction}

For patients suffering from ST-elevation myocardial infarction (STEMI), timely revascularization with primary percutaneous coronary intervention (pPCI) of the infarct-related artery (IRA) is the treatment of choice. Of these patients, multivessel disease (MVD) identified angiographically upon intervention of the culprit lesion is common, with some studies suggesting a prevalence of approximately $50 \%[1,2]$. MVD in patients with STEMI
Correspondence to:

Joshua H. Arnold, joshuaarnold@mail.tau.ac.il 
has been shown to be a predictor of short- and long-term mortality rates as well as reinfarction when compared to patients with single-vessel disease [3-5]. Whether these patients would benefit from intervention of non-IRA has long been controversial $[6,7]$. Recently, data from the COMPLETE trial showed a significantly reduced risk of cardiovascular events when complete revascularization of STEMI patients with MVD is achieved [8]. Other smaller randomized studies have also suggested reduced adverse cardiac events for patients receiving complete revascularization, including the Cvlprit [9], PRAMI [10], DANAMI-3-PRIMULTI [11], and Compare-Acute trials [12]. Furthermore, a recent meta-analysis of available randomized trials comparing multivessel versus culprit only revascularization found similar reduction in risk of reinfarction [13].

Studies have shown patients are at increased risk of ischemic events [14-16] and mortality $[17,18]$ when onetime complete revascularization is attempted compared to staged PCI (sPCI), suggesting that sPCI is the safer method for addressing nonculprit lesions [19]. However, what is considered a "reasonable time" following pPCI until completion of coronary revascularization is still a matter of great uncertainty and debate. Because there are conflicting data on this issue [20], the need for clear guidelines for optimal management of these patients is vital. Whether it is necessary to revascularize non-IRA as soon as possible or to delay procedures remains controversial $[10,21]$. Thus, we aimed to evaluate the rate of major adverse cardiac events (MACE) over time for STEMI patients with MVD undergoing pPCI and whether patients are at increased risk of adverse outcomes as time between the procedures lengthens.

\section{Materials and Methods}

\section{Patients and Setting}

Since 2004, all consecutive STEMI cases at the Rabin Medical Center, Israel, have been prospectively entered into a comprehensive registry for purposes of monitoring patient-related parameters and clinical events. This study is therefore based on clinical data of consecutive patients presenting with STEMI and treated by pPCI between January 2004 and January 2018. The Rabin Medical Center includes 2 campuses: the Beilinson and Hasharon medical centers. Of the 3,002 patients in the registry, 1,555 were eligible for the study: patients who were found to have a nonculprit lesion, defined as the presence of at least 1 angiographically significant lesion that was amenable to successful treatment with PCI and was located in a vessel with a diameter of at least $2.5 \mathrm{~mm}$. Lesions were considered angiographically significant if they were associated with at least $70 \%$ stenosis of the vessel diameter on visual estimation (e.g., taken during the pPCI angiography) or with moderate stenosis accompanied by a fractional flow reserve measurement of 0.80 or less at the time of sPCI.

Patients were divided into quartiles of duration from $\mathrm{pPCI}$ to sPCI: 0-7 days (Q1), 7-22 days (Q2), 22-42 days (Q3), and $>42$ days (Q4). We excluded all patients who were treated for their nonculprit artery during the index procedure or sent to coronary bypass surgery (CABG) for revascularization of the non-IRA. We also excluded those who were treated urgently for the nonculprit artery due to an acute coronary syndrome during their wait for a scheduled sPCI. Other exclusion criteria were presentation with cardiogenic shock during STEMI, treatment by thrombolysis ( $<1 \%$ of patients), and ineligibility for year-long dual antiplatelet pharmacotherapy. The study and registry were approved by the Institutional Review Boards of the medical center.

\section{Interventional Procedure}

All patients provided explicit written informed consent before undergoing cardiac catheterization. Pretreatment consisted of aspirin $300 \mathrm{mg}$ and unfractionated heparin $(70 \mathrm{U} / \mathrm{kg})$; clopidogrel 300 or $600 \mathrm{mg}$, prasugrel $60 \mathrm{mg}$, or ticagrelor $180 \mathrm{mg}$ was administered as a loading dose before or immediately after pPCI. The utilization of glycoprotein IIb/IIIa inhibitors and the choice of the type of coronary stent and other adjunct therapy were left to the discretion of the primary operator. All stents were implanted with moderate-to-high deployment pressure (12-16 atm). All patients received dual antiplatelet therapy with aspirin $100 \mathrm{mg}$ daily and a P2Y12 inhibitor (clopidogrel, prasugrel, or ticagrelor) for at least 12 months after the sPCI according to STEMI guidelines directed management.

\section{Study End Points}

Immediate and in-hospital clinical events were prospectively recorded in the institutional database. During the follow-up, patients completed standardized questionnaires on clinical events at 6-month intervals either by telephone or in the outpatient clinic. When indicated, records from peripheral hospitals were acquired to verify the data. All events were further confirmed and adjudicated by the institutional clinical events adjudication committees. Survival status was assessed by municipal civil registries after 1 and 2 years.

Primary outcomes included all-cause mortality and MACE following sPCI, at the median time of each quartile which comprised death, repeat myocardial infarction (MI), need for target vessel revascularization (TVR), and/or CABG. Renal failure was defined as glomerular filtration rate below $50 \mathrm{~mL} / \mathrm{min} / 1.73 \mathrm{~m}^{2}$ (calculated according to the Modification of Diet in Renal Disease formula). Anemia was defined as hemoglobin levels lower than $13.0 \mathrm{~g} / \mathrm{dL}$ for men and $12.0 \mathrm{~g} / \mathrm{dL}$ for women.

\section{Statistical Analysis}

Continuous data are summarized as mean and standard deviation, or median and interquartile range, and categorical data as frequency (\%). Student $t$ test or analysis of variance was used to compare continuous variables between groups, and $\chi^{2}$ or Fisher's exact test was used for categorical variables. The normality of variable distributions was assessed using the Kolmogorov-Smirnov test. Time-to-event curves were constructed using the Kaplan-Meier method and compared using log-rank test. Stepwise variable selection of significant univariate predictors $(p<0.1)$ was used to identify variables for inclusion in the multivariate model. Cox regression analysis was performed to determine independent predictors of the primary end point, accounting for known baseline cardiovascular risk differences, 
Table 1. Baseline characteristics

\begin{tabular}{|c|c|c|c|c|c|c|}
\hline & Total $(n=1,555)$ & $\mathrm{Q} 1(n=423)$ & $\mathrm{Q} 2(n=382)$ & Q3 $(n=379)$ & $\mathrm{Q} 4(n=371)$ & $p$ value \\
\hline Age, years & $65.7 \pm 11.5$ & $64.7 \pm 12.0$ & $65.7 \pm 11.8$ & $65.7 \pm 10.8$ & $67.1 \pm 11.1$ & 0.368 \\
\hline Gender, $\%$ female & 21.4 & 22.2 & 18.8 & 20.1 & 24.5 & 0.240 \\
\hline $\mathrm{DM}, \%$ & 46.3 & 39.5 & 44.2 & 47.2 & 55.3 & $<0.001$ \\
\hline Hypertension, \% & 76.8 & 70.9 & 70.9 & 80.2 & 86.0 & $<0.001$ \\
\hline Smoking, \% & 37.8 & 41.6 & 35.9 & 37.2 & 36.1 & 0.294 \\
\hline $\mathrm{CHF}, \%$ & 10.4 & 10.2 & 10.5 & 7.4 & 13.5 & 0.057 \\
\hline PVD, $\%$ & 3.5 & 1.7 & 3.1 & 3.2 & 6.2 & 0.005 \\
\hline CVA, \% & 6.8 & 6.6 & 6.8 & 5.8 & 7.8 & 0.938 \\
\hline Atrial fibrillation, $\%$ & 33.6 & 35.8 & 32.1 & 30.9 & 34.6 & 0.001 \\
\hline Malignancy, \% & 11.1 & 10.2 & 9.9 & 11.3 & 13.2 & 0.462 \\
\hline Dementia, \% & 1.7 & 1.9 & 1.6 & 1.1 & 2.2 & 0.671 \\
\hline CABG, $\%$ & 6.6 & 3.3 & 7.1 & 6.1 & 10.5 & 0.001 \\
\hline
\end{tabular}

CABG, coronary artery bypass graft surgery; CHF, congestive heart failure; COPD, chronic obstructive pulmonary disorder; CVA, cerebrovascular accident; DM, diabetes mellitus; EF, ejection fraction; eGFR, estimated glomerular filtration rate; PVD, peripheral vascular disease.

Table 2. Procedural characteristics for pPCI

\begin{tabular}{|c|c|c|c|c|c|c|}
\hline & Total $(n=1,555)$ & $\mathrm{Q} 1(n=423)$ & Q2 $(n=382)$ & Q3 $(n=379)$ & $\mathrm{Q} 4(n=371)$ & $p$ value \\
\hline Number of diseased vessels & 2.18 & 2.24 & 2.15 & 2.13 & 2.20 & 0.198 \\
\hline Radial approach, \% & 52.1 & 50.3 & 52.8 & 52.7 & 52.0 & 0.270 \\
\hline Bifurcation, $\%$ & 17.7 & 23.0 & 18.0 & 15.5 & 12.8 & 0.325 \\
\hline Left main PCI, \% & 3.7 & 3.8 & 2.6 & 4.0 & 4.3 & 0.630 \\
\hline Anterior AMI, \% & 47.2 & 47.8 & 45.6 & 46.3 & 49.3 & 0.439 \\
\hline Venous graft AMI, \% & 4.5 & 2.4 & 3.3 & 3.7 & 4.1 & 0.126 \\
\hline Calcification, $\%$ & 13.4 & 16.8 & 11.3 & 15.8 & 8.4 & 0.003 \\
\hline Long lesion, $\%$ & 26.8 & 26.2 & 26.1 & 27.7 & 26.0 & 0.286 \\
\hline GP2B3A inhibitors, \% & 2.7 & 2.4 & 3.1 & 3.0 & 2.8 & 0.231 \\
\hline IABP, $\%$ & 1.2 & 1.2 & 1.1 & 1.4 & 1.3 & 0.430 \\
\hline Clopidogrel, \% & 74.8 & 71.3 & 72.3 & 73.9 & 81.7 & 0.078 \\
\hline Prasugrel, \% & 18.2 & 23.3 & 18.6 & 21.0 & 15.6 & 0.082 \\
\hline
\end{tabular}

AMI, acute myocardial infarction; CTO, chronic total occlusion; IABP, intra-aortic balloon pump; NOAC, nonvitamin K antagonist oral anticoagulants; pPCI, primary percutaneous coronary intervention; VKA, vitamin K antagonist.

which included: age, sex, diabetes mellitus, renal failure, hypertension, obesity, peripheral vascular disease, and cerebrovascular accident. Effect sizes are presented as hazard ratio (HR) and 95\% confidence interval (CI). As a secondary analysis, propensity score was then computed using a multivariable logistic regression model with receipt of patients treated after the median (over 22 days) 3 versus controls as the independent variable and all pre-PCI and intraprocedural variables as covariates. The propensity score matching was performed using a "closest neighbor, greedy" algorithm, attempting to match to each patient above the median time to sPCI with a cohort 


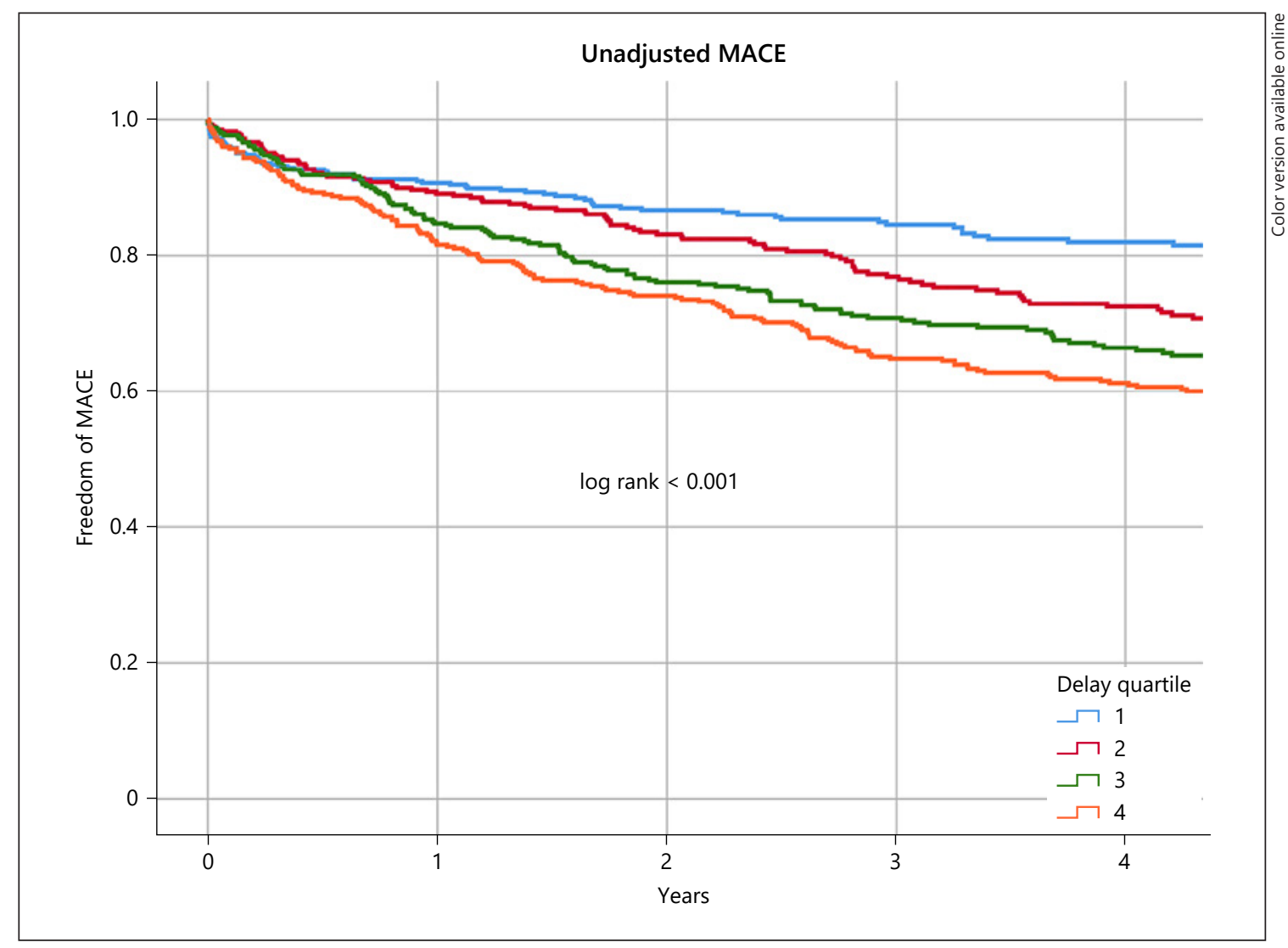

Fig. 1. Kaplan-Meier curves showing unadjusted rates of MACEs for each delayed PCI quartile group of patients. PCI, percutaneous coronary intervention; MACEs, major adverse cardiac events.

patient from the rest of the cohort with the closest propensity score. To control for adequate matching, we also calculated the standardized differences of means and of dichotomous variable distributions for the covariates used for the matching. We regarded a cutoff value of $\pm 0.96 \times[\sqrt{ }(2 / n)]$ to assume the prevalence of a covariate is equal between 2 groups with equal number of subjects. All standardized differences were below that cutoff value (see online suppl. Table 1; see www.karger.com/doi/10.1159/000517295 for all online suppl. material). Each pair was used once. Unpaired patients were discarded from the analysis. The propensity score was then used to select 296 well-matched pairs of patients in each group (delayed sPCI $=296$ vs. early $\mathrm{sPCI}=296$ ) were selected. All statistical analyses were performed with IBM SPSS V. 27. A $p$ value of $<0.05$ was considered statistically significant.

\section{Results}

In total, 1,555 patients were included in our analysis. The average age at presentation was 65.7. $\pm 11.5,333$ were female patients $(21.4 \%)$. The average time between pPCI and sPCI was 28.3 days ( \pm 24.8$) .46 .3 \%$ had diabetes mel- litus, $76.8 \%$ had hypertension, $10.4 \%$ were diagnosed with heart failure, and $3.5 \%$ had a previous diagnosis of peripheral vascular disease (PVD). Patients were separated into quartiles based on days elapsed following pPCI until day of sPCI: quartile 1 (Q1) 0-7 days (423 patients), quartile 2 (Q2) 7-22 days (382 patients), quartile $3(\mathrm{Q} 3)$ $22-41$ days (379 patients), and quartile 4 (Q4) $>42$ days (371 patients). Differences in rates of diabetes $(p<0.001)$, hypertension $(p<0.001)$, PVD $(p=0.005)$, and prior CABG $(p=0.001)$ existed between the quartiles (Tables $1,2)$.

Patients in Q1 experienced 70 MACE (16.5\%), patients in Q2 experienced 81 MACE (21.2\%), patients in Q3 experienced 98 MACE (25.8\%), and patients in Q4 experienced 112 MACE (30.1\%) (log-rank $<0.001$, Fig. 1$)$. When unadjusted rates of MACE were compared between all groups, all MACE outcome components were significantly associated with increasing time between pPCI and sPCI, including: re-MI $(p=0.008)$, TVR $(p<0.001)$, CABG $(p=0.041)$, and all-cause death $(p<0.001)$ (online 
suppl. Table 2). Differences in unadjusted all-cause mortality rates were statistically significant $(p<0.001)$ between the quartiles (online suppl. Fig. 1).

Multivariate cox regression analysis was performed, adjusting for variables such as comorbid conditions between the quartiles. This analysis revealed delayed sPCI as an independent factor for risk of MACE ( $\mathrm{HR}=1.226$ [95\% CI: $1.129-1.331, p<0.001])$. Other factors associated with increased risk of MACE included: age at PCI $(\mathrm{HR}=1.015$ [95\% CI: 1.005-1.025, $p=0.002])$, history of diabetes mellitus ( $\mathrm{HR}=1.272$ [95\% CI 1.050-1.541, $p=$ $0.014]$ ), decreased estimated ejection fraction ( $\mathrm{HR}=$ 0.978 [95\% CI $0.968-0.988 p<0.001]$ ), and renal insufficiency $(\mathrm{HR}=2.260$ [95\% CI 1.823-2.801, $p<0.001])$ (Table 3). A multivariate cox regression analysis assessing death as the primary end point revealed a nonsignificant association $(\mathrm{HR}=1.022$ [95\% CI: 0.925-1.129, $p=0.671])$ with delay length. Patient age at PCI $(\mathrm{HR}=1.043 ; 1.03-$ $1.056, p<0.001)$, history of diabetes mellitus $(\mathrm{HR}=1.402$ [95\% CI: 1.102-1.784, $p=0.006])$, prior PVD $(\mathrm{HR}=1.768$ [95\% CI: $1.133-2.758, p=0.012])$, decreased estimated ejection fraction $(\mathrm{HR}=0.974$ [95\% CI 0.962-0.986 $p<$ $0.001]$ ), renal insufficiency ( $\mathrm{HR}=2.759$ [95\% CI 2.134$3.568, p<0.001])$, and prior stroke ( $\mathrm{HR}=1.501[95 \% \mathrm{CI}$ $1.076-2.092, p=0.017]$ ) were all found to be associated with increased risk of death (online suppl. Table 3).

We then performed a secondary analysis, following propensity matching, which compared those treated before and after the median time ( 22 days) between pPCI and sPCI, again revealing delayed sPCI as an independent factor for risk of MACE (HR = 1.503 [95\% CI: 1.1921.896, $p=0.001]$ ) (Fig. 2). Other factors associated with increased risk of MACE included: congestive heart failure $(\mathrm{HR}=1.976$ [95\% CI: 1.525-2.561, $p<0.001])$, prior PVD $(\mathrm{HR}=1.635$ [95\% CI: $1.092-2.450, p=0.017])$, and renal insufficiency $(\mathrm{HR}=2.034$ [95\% CI: 1.604-2.580, $p<$ 0.001]) (Table 4). When mortality was assessed as the end point, delayed sPCI was not found to be a significant independent risk factor ( $\mathrm{HR}=1.163$ [95\% CI: 0.890-1.521, $p=0.269$, online suppl. Table 4 and Fig. 3$]$ ).

\section{Discussion}

The goal of the current study is to better understand the effects of timing between primary intervention of culprit lesions in STEMI patients and staged interventions of nonculprit lesions. With growing trends toward complete revascularization of patients with MVD, the need for accurate information on the optimal timing of revascular-
Table 3. Cox regression MACE

\begin{tabular}{lllr}
\hline & HR & Range & $p$ value \\
\hline Age, years & 1.015 & $1.005-1.025$ & 0.002 \\
Gender (female) & 1.064 & $0.862-1.314$ & 0.561 \\
DM & 1.272 & $1.050-1.541$ & 0.014 \\
HTN & 1.049 & $0.801-1.375$ & 0.727 \\
CHF & 1.161 & $0.908-1.375$ & 0.234 \\
PVD & 1.373 & $0.923-2.044$ & 0.118 \\
Estimated EF & 0.978 & $0.968-0.988$ & $<0.001$ \\
eGFR <60 & 2.260 & $1.823-2.801$ & $<0.001$ \\
Prior stroke & 1.227 & $0.911-1.654$ & 0.178 \\
Radial & 1.070 & $0.845-1.354$ & 0.575 \\
Time between quartiles & 1.226 & $1.129-1.331$ & $<0.001$ \\
\hline
\end{tabular}

CHF, congestive heart failure; DM, diabetes mellitus; EF, ejection fraction; eGFR, estimated glomerular filtration rate; HTN, hypertension; PVD, peripheral vascular disease; MACE, major adverse cardiovascular event; $\mathrm{HR}$, hazard ratio.

ization is necessary. A clearly defined protocol for management has not emerged due to the lack of robust evidence and conflicting data. Our study showed that increased time to staged intervention of nonculprit lesions was associated with higher rates of MACE.

The ESC/EACTS guidelines on myocardial revascularization highlight a lack of evidence on optimal timing of staged procedures [14]. When considering the proper timing for sPCI, the clinician must consider many variables, including risk of recurrent ischemic events, contrast exposure, severity of initial infarction, patient symptoms, and other comorbidities. Two recent studies have shown improved outcomes and reduction of MACE for patients with MVD receiving SPCI within 1-2 weeks of initial presentation $[22,23]$. Other studies suggest that a more conservative approach with longer wait times $(>3$ weeks) can improve outcomes [24]. A sub-analysis of the COMPLETE trial assessing timing showed consistent improvement in outcomes irrespective of timing of secondary interventions [25], and a recent meta-analysis similarly demonstrated consistent benefits for STEMI patients regardless of whether the complete revascularization was achieved during the index procedure or as a staged intervention ( $\leq 45$ days) [26].

In this study, the risk for MACE increased as duration between primary and staged procedures increased. This may be due to the fact that patients with non-IRA coronary artery disease are inherently at increased risk for adverse events and mortality [4], and these events were a manifestation of this burden over time. Other studies have 


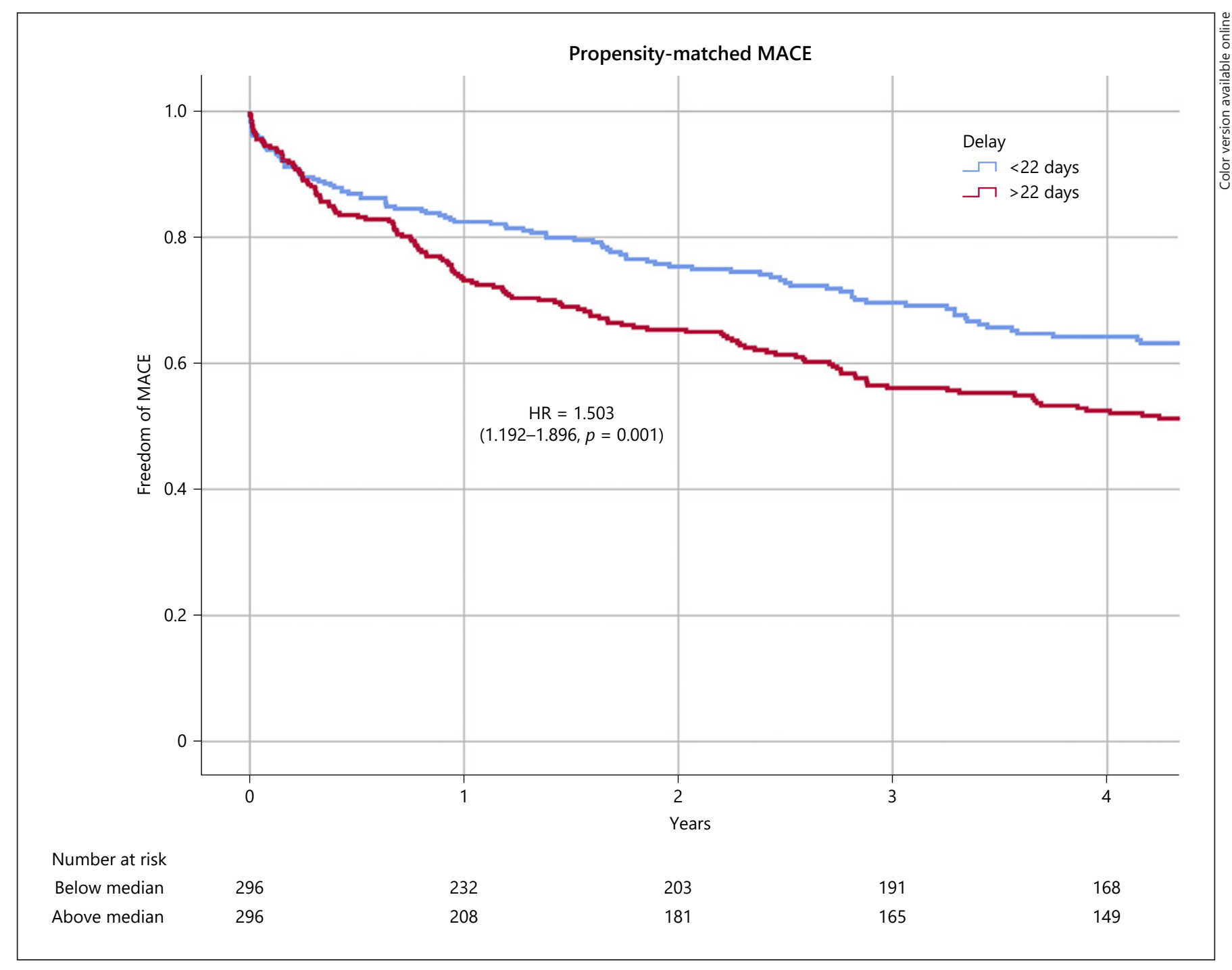

Fig. 2. Kaplan-Meier curves showing rates of MACEs for sPCI groups following propensity matching. sPCI, staged percutaneous coronary intervention; HR, hazard ratio; MACEs, major adverse cardiovascular events.

shown that complex coronary plaques [27] and concomitant plaque ruptures $[28,29]$ were already present in nonculprit arteries at presentation of STEMI, increasing the urgency of revascularization of high-risk plaques. Patients within our dataset who had their secondary interventions delayed had significantly more comorbidities. Despite our adjustment for available comorbidities, these patients may have experienced higher rates of MACE because they had a larger burden of coronary artery disease and/or comorbid conditions contributing to the likelihood of adverse events. This may have also contributed to the clinician's decision to delay sPCI. However, when both Cox regression and propensity matching were done within each quartile for isolation of timing as an independent variable, delayed sPCI remained a risk factor for MACE.

The consideration of timing is inherent in decisionmaking when clinicians are presented with patients having STEMI and MVD. Studies discuss the decreased or similar risk of adverse effects $[19,30,31]$ if flow is restored within nonculprit lesions during the index procedure, while others show planning a separate intervention to be more efficacious [14-17]. Potential benefits of sPCIs include time to properly assess complex nonculprit lesions with noninvasive testing and fractional flow reserve [21], as well as a proposed time for myocardial recovery [17]. Of consideration, revascularization of non- 
Table 4. Cox regression propensity matched MACE

\begin{tabular}{lllr}
\hline & HR & Range & $p$ value \\
\hline Age, years & 1.009 & $0.996-1.021$ & 0.187 \\
DM & 1.183 & $0.922-1.518$ & 0.186 \\
HTN & 0.801 & $0.526-1.221$ & 0.302 \\
CHF & 1.976 & $1.525-2.561$ & $<0.001$ \\
PVD & 1.635 & $1.092-2.450$ & 0.017 \\
Estimated EF & 0.990 & $0.978-1.002$ & 0.105 \\
eGFR <60 & 2.034 & $1.604-2.580$ & $<0.001$ \\
Gender factor & 0.520 & 0.2061 .315 & 0.167 \\
Median time to sPCI & 1.503 & $1.191-1.896$ & 0.001 \\
\hline
\end{tabular}

$\mathrm{CHF}$, congestive heart failure; EF, ejection fraction; eGFR, estimated glomerular filtration rate; HTN, hypertension; sPCI, staged percutaneous coronary intervention; PVD, peripheral vascular disease; MACE, major adverse cardiovascular event; HR, hazard ratio.

culprit arteries soon after STEMI, either during the index procedure or during an early sPCI, may have added risk due to the fact that the myocardium is undergoing remodeling. This must be weighed against complications associated with performing a duplicate procedure such as increased contrast burden and nephropathy risk [32], a potentially higher incidence of procedural complications from higher patient loads of anticoagulant and antithrombotic therapies in early sPCI [17], as well as the inherent risk associated with prolonged patient exposure to vulnerable plaque burden when intervention is delayed.

Following infarction, a multifactorial process involving inflammatory mediators, the renin-angiotensin-aldosterone system, and the sympathomimetic nervous system causes myocyte hypertrophy and dilation [33]. Noninfarcted areas that surround the infarcted lesion are under increased stress, due to a multitude of factors, and undergo a detrimental form of hypertrophy [34]. Furthermore, the plaques harbored in non-IRA obstructive lesions have been found to be, more often than not, of vulnerable plaque morphology (thin-cap fibroatheroma) as detected by optical coherence tomography [35] which have been most commonly implicated as the cause of acute coronary events $[36,37]$. The presence of thin-cap fibroatheroma lesions as detected by optical coherence tomography can also be predictive of postprocedural MI occurrence, further strengthening the need to intervene in such lesions [38]. This may be compounded in patients with MVD and perhaps could be mitigated by intervening on non-IRA lesions, which could be suggested by other studies showing improved short-term [39] and long-term [40] outcomes for patients who undergo complete revascularization more urgently. If patients require secondary procedures to intervene on non-IRA, those with an increased burden of comorbidities should be evaluated sooner and undergo early revascularization.

\section{Study Limitations}

One main limitation of this study is that it is an observational, single-center, and post hoc analysis of our cohort. This is an initial hypothesis-generating study. It is not conclusive or adequate to change practice per se. Although based on an all-comer prospective registry, biases may have influenced the selection of data, the method of therapy and other factors associated with outcome, although our primary outcome measures remained significant after multivariate analysis. The observational nature of the study weakens our ability to report on a causal relationship between time duration between the index event and sPCI and outcomes. Patient baseline characteristics also significantly differed between quartiles; nevertheless, after multivariate analysis, delayed sPCI remained a significant risk factor for MACE. In this large real-world registry, our data support an early approach to sPCI in patients suffering from STEMI presenting with MVD.

\section{Conclusions}

Patients who presented with STEMI and MVD and who underwent complete revascularization were at increased risk of experiencing MACE with increased time interval between primary intervention and staged intervention. These data should be taken into account when treating patients with STEMI and MVD using the staged approach geared toward complete coronary revascularization.

\section{Statement of Ethics}

The Institutional Review Board for Rabin Medical Center approved this process prior to beginning the data collection. All patients gave written informed consent prior to participation. The IRB reference number is RMC-3741.

\section{Conflict of Interest Statement}

The authors have no conflicts of interest to declare. 


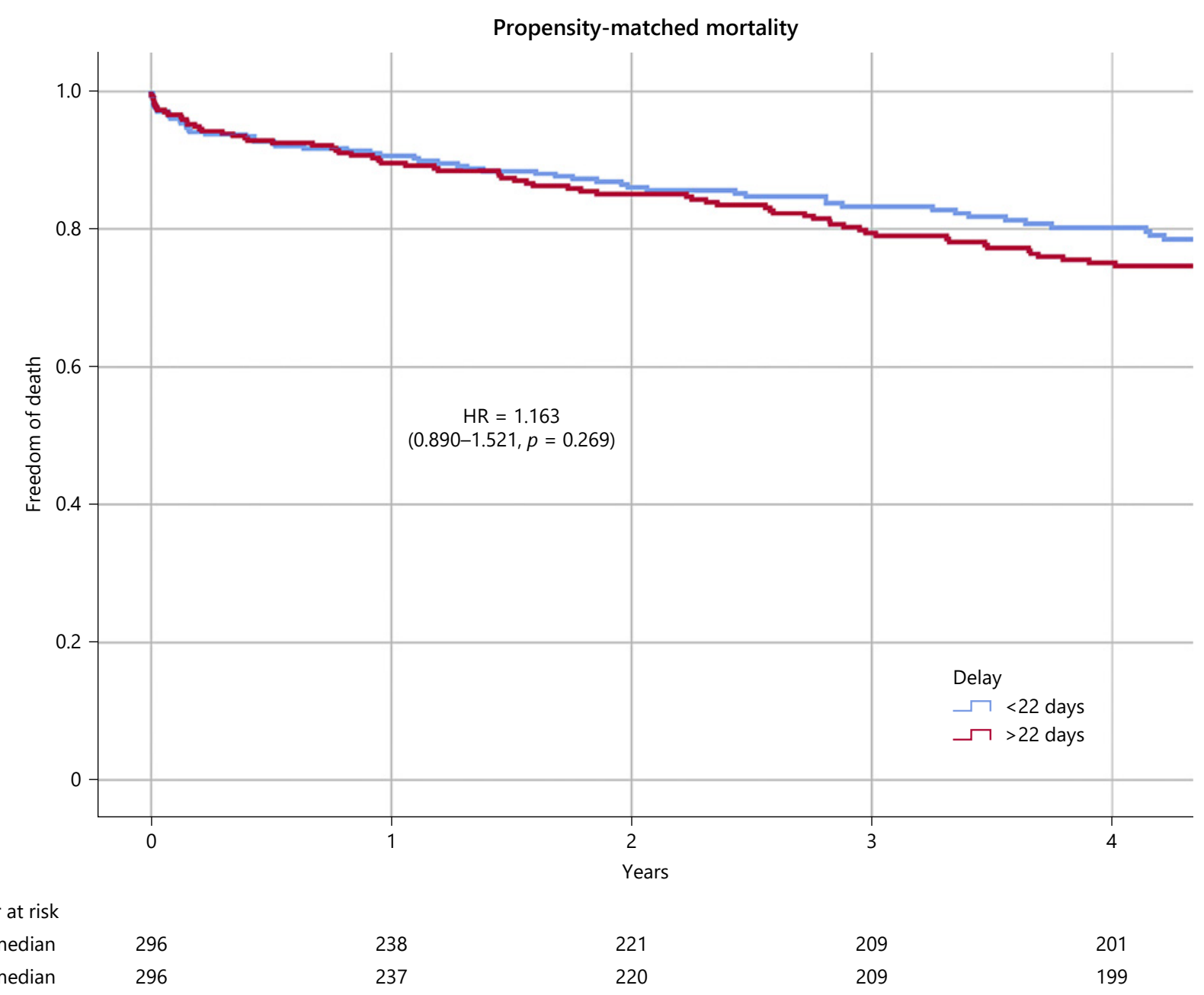

Fig. 3. Kaplan-Meier curves showing rates of mortality over time for sPCI groups following propensity matching. sPCI, staged percutaneous coronary intervention; HR, hazard ratio.

\section{Funding Sources}

The authors have no funding sources to declare.

\section{Author Contributions}

All authors fulfill the ICJME criteria for authorship. J.H.A was responsible for the writing, conception, and revision of the manuscript. T.B. was responsible for data analysis and acquisition. G.G. and H.V.A. were responsible for critical revision of the manu- script. R.K. was responsible for the conception and revision of the manuscript, and L.P. was responsible for the conception and design, data analysis, and revision of the manuscript. All the authors have read and approved the final version of this work.

\section{Data Availability Statement}

All data generated or analyzed during this study are not publicly available on legal or ethical grounds. Further inquiries can be directed to the corresponding author. 


\section{References}

1 Park DW, Clare RM, Schulte PJ, Pieper KS, Shaw LK, Califf RM, et al. Extent, location, and clinical significance of non-infarct-related coronary artery disease among patients with ST-elevation myocardial infarction. JAMA. 2014 Nov 19;312(19):2019-27.

2 Dziewierz A, Siudak Z, Rakowski T, Zasada W, Dubiel JS, Dudek D. Impact of multivessel coronary artery disease and noninfarct-related artery revascularization on outcome of patients with ST-elevation myocardial infarction transferred for primary percutaneous coronary intervention (from the EUROTRANSFER Registry). Am J Cardiol. 2010;106:342.

3 Halkin A, Singh M, Nikolsky E, Grines CL, Tcheng JE, Garcia E, et al. Prediction of mortality after primary percutaneous coronary intervention for acute myocardial infarction: the CADILLAC risk score. J Am Coll Cardiol. 2005;45:1397-405.

4 Sorajja P, Gersh BJ, Cox DA, McLaughlin MG, Zimetbaum P, Costantini C, et al. Impact of multivessel disease on reperfusion success and clinical outcomes in patients undergoing primary percutaneous coronary intervention for acute myocardial infarction. Eur Heart J. 2007;28:1709-16.

5 Muller DWM, Topol EJ, Ellis SG, Sigmon KN, Lee K, Califf RM. Multivessel coronary artery disease: a key predictor of short-term prognosis after reperfusion therapy for acute myocardial infarction. Am Heart J. 1991;121: 1042-9.

6 Wood DA, Cairns JA, Mehta SR. Multivessel revascularization and ST-segment elevation myocardial infarction: do we have the complete answer? Circ Cardiovasc Interv. 2017; 10(4):e005215.

7 Vogel B, Mehta SR, Mehran R. Reperfusion strategies in acute myocardial infarction and multivessel disease. Nat Rev Cardiol. 2017;14: 665-78.

8 Mehta SR, Wood DA, Storey RF, Mehran R, Bainey KR, Nguyen H, et al. Complete revascularization with multivessel PCI for myocardial infarction. N Engl J Med. 2019 Oct 10; 381(15):1411-21.

9 Gershlick AH, Khan JN, Kelly DJ, Greenwood JP, Sasikaran T, Curzen N, et al. Randomized trial of complete versus lesion-only revascularization in patients undergoing primary percutaneous coronary intervention for STE$\mathrm{MI}$ and multivessel disease: the CvLPRIT trial. J Am Coll Cardiol. 2015;65:963.

10 Wald DS, Morris JK, Wald NJ, Chase AJ, Edwards RJ, Hughes LO, et al. Randomized trial of preventive angioplasty in myocardial infarction. N Engl J Med. 2013;369:1115-23.

11 Engstrom T, Kelbaek H, Helqvist S, Hofsten DE, Klovgaard L, Holmvang L, et al. Complete revascularisation versus treatment of the culprit lesion only in patients with ST-segment elevation myocardial infarction and multivessel disease (DANAMI-3-PRI-
MULTI): an open-label, randomized controlled trial. Lancet. 2015;386:665-71.

12 Smits PC, Abdel-Wahab M, Neumann FJ, Boxma-de Klerk BM, Lunde K, Schotborgh $\mathrm{CE}$, et al. Fractional flow reserve-guided multivessel angioplasty in myocardial infarction. N Engl J Med. 2017;376(13):1234-44.

13 Atti V, Gwon Y, Narayanan MA, Garcia S, Sandoval Y, Brilakis ES, et al. Multivessel versus culprit-only revascularization in STEMI and multivessel coronary artery disease: meta-analysis of randomized trials. JACC Cardiovasc Interv. 2020;13(13):1571-82.

14 Neumann FJ, Sousa-Uva M, Ahlsson A, Alfonso F, Banning AP, Benedetto U, et al. 2018 ESC/EACTS Guidelines on myocardial revascularization. Eur Heart J. 2018-2019 Jan 7; 40(2):87-165

15 Politi L, Sgura F, Rossi R, Monopoli D, Guerri E, Leuzzi C, et al. A randomised trial of target-vessel versus multi-vessel revascularization in ST-elevation myocardial infarction: major adverse cardiac events during longterm follow-up. Heart. 2010;96:662-7.

16 Di Mario C, Mara S, Flavio A, Imad S, Antonio $M$, Anna $P$, et al. Single vs. multivessel treatment during primary angioplasty: results of the multicentre randomised HEpacoat for cuLPrit or multivessel stenting for acute myocardial infarction (HELP AMI) study. Int J Cardiovasc Intervent. 2004;6:128-33.

17 Li Z, Zhou Y, Xu Q, Chen X. Staged versus one-time complete revascularization with percutaneous intervention in STEMI patients with multivessel disease: a systemic review and meta-analysis. PLoS One. 2017 Jan 20; 12(1):e0169406.

18 Kornowski R, Mehran R, Dangas G, Nikolsky E, Assali A, Claessen BE, et al. Prognostic impact of staged versus "one-time" multivessel percutaneous intervention in acute myocardial infarction: analysis from the HORIZONS-AMI (harmonizing outcomes with revascularization and stents in acute myocardial infarction) trial. J Am Coll Cardiol. 2011; 58(7):704-11.

19 Bates ER, Tamis-Holland JE, Bittl JA, O’Gara PT, Levine GN. PCI strategies in patients with ST-segment elevation myocardial infarction and multivessel coronary artery disease. J Am Coll Cardiol. 2016;68(10):1066-81.

20 Gaffar R, Habib B, Filion KB, Reynier P, Eisenberg MJ. Optimal timing of complete revascularization in acute coronary syndrome: a systematic review and meta-analysis. J Am Heart Assoc. 2017 Apr 10;6(4):e005381.

21 Saito Y, Kobayashi Y. Percutaneous coronary intervention strategies in patients with acute myocardial infarction and multivessel disease: completeness, timing, lesion assessment, and patient status. J Cardiol. 2019 Aug;74(2): 95-101.

22 Zhao XD, Zhao GQ, Wang X, Shi ST, Zheng W, Guo RF, et al. Optimal timing of staged percutaneous coronary intervention in ST- segment elevation myocardial infarction patients with multivessel disease. J Geriatr Cardiol. 2018 May;15(5):356-62.

23 Fukutomi M, Toriumi S, Ogoyama Y, Oba Y, Takahashi M, Funayama H, et al. Outcome of staged percutaneous coronary intervention within two weeks from admission in patients with ST-segment elevation myocardial infarction with multivessel disease. Catheter Cardiovasc Interv. 2019 Apr 1;93(5):E262-268.

24 Lee WC, Wu BJ, Fang CY, Chen CJ, Yang CH, Yip HK, et al. Timing of staged percutaneous coronary intervention for a non-culprit lesion in patients with anterior wall ST segment elevation myocardial infarction with multiple vessel disease. Int Heart J. 2016 Jul 27;57(4): 417-23.

25 Wood DA, Cairns JA, Wang J, Mehran R, Storey $\mathrm{RF}$, Nguyen $\mathrm{H}$, et al. Timing of staged nonculprit artery revascularization in patients with ST-segment elevation myocardial infarction: COMPLETE trial. J Am Coll Cardiol. 2019 Dec 3;74(22):2713-23.

26 Bainey KR, Engstrøm T, Smits PC, Gershlick AH, James SK, Storey RF, et al. Complete vs. culprit-lesion-only revascularization for STsegment elevation myocardial infarction: a systematic review and meta-analysis. JAMA Cardiol. 2020 May 20;5(8):1-9.

27 Goldstein JA, Demetriou D, Grines CL, Pica M, Shoukfeh M, O’Neill WW. Multiple complex coronary plaques in patients with acute myocardial infarction. N Engl J Med. 2000 Sep 28;343(13):915-22.

28 Hong MK, Mintz GS, Lee CW, Kim YH, Lee SW, Song JM, et al. Comparison of coronary plaque rupture between stable angina and acute myocardial infarction: a three-vessel intravascular ultrasound study in 235 patients. Circulation. 2004 Aug 24;110(8):928-33.

29 Fukunaga M, Fujii K, Nakata T, Shibuya M, Miki K, Kawasaki D, et al. Multiple complex coronary atherosclerosis in diabetic patients with acute myocardial infarction: a three-vessel optical coherence tomography study. EuroIntervention. 2012 Dec 20;8(8):955-61.

30 Pasceri V, Patti G, Pelliccia F, Gaudio C, Speciale G, Mehran R, et al. Complete revascularization during primary percutaneous coronary intervention reduces death and myocardial infarction in patients With multivessel disease: meta-analysis and meta-regression of randomized trials. JACC Cardiovasc Interv. 2018 May 14;11(9):833-43.

31 Hlinomaz O, Groch L, Polokova L, Lehar F, Vekov T, Griva M, et al. Multivessel disease diagnosed at the time of primary PCI for STEMI: complete revascularization versus conservative strategy. Eur Heart J. 2015 Aug 1;36: 825 .

32 Marenzi G, Lauri G, Assanelli E, Campodonico J, De Metrio M, Marana I, et al. Contrastinduced nephropathy in patients undergoing primary angioplasty for acute myocardial infarction. J Am Coll Cardiol. 2004;44:1780-5. 
33 Sutton MG, Sharpe N. Left ventricular remodeling after myocardial infarction: pathophysiology and therapy. Circulation. 2000; 101(25):2981-8.

34 French BA, Kramer CM. Mechanisms of post-infarct left ventricular remodeling. Drug Discov Today Dis Mech. 2007;4(3):185-96.

35 Pinilla-Echeverri N, Mehta SR, Wang J, Lavi S, Schampaert E, Cantor WJ, et al. Nonculprit lesion plaque morphology in patients with ST-segment-elevation myocardial infarction: results from the COMPLETE trial optical coherence tomography substudys. Circ Cardiovasc Interv. 2020;13(7):e008768.
36 Davies MJ. A macro and micro view of coronary vascular insult in ischemic heart disease. Circulation. 1990;82(Suppl 3):II38-46.

37 Virmani R, Kolodgie FD, Burke AP, Farb A, Schwartz SM. Lessons from sudden coronary death: a comprehensive morphological classification scheme for atherosclerotic lesions. Arterioscler Thromb Vasc Biol. 2000;20(5): 1262-75.

38 Lee T, Yonetsu T, Koura K, Hishikari K, Murai $\mathrm{T}$, Iwai $\mathrm{T}$, et al. Impact of coronary plaque morphology assessed by optical coherence tomography on cardiac troponin elevation in patients with elective stent implantation. Circ Cardiovasc Interv. 2011;4(4):378-86.
39 Marino M, Crimi G, Leonardi S, Ferlini M, Repetto A, Camporotondo R, et al. Comparison of outcomes of staged complete revascularization versus culprit lesion-only revascularization for ST-elevation myocardial infarction and multivessel coronary artery disease. Am J Cardiol. 2017;119(4):508-14.

40 Lu C, Huang H, Li J, Zhao J, Zhang Q, Zeng $Z$, et al. Complete versus culprit-only revascularization during primary percutaneous coronary intervention in ST-elevation myocardial infarction patients with multivessel disease: a meta-analysis. Kaohsiung J Med Sci. 2013; 29(3):140-9. 